\title{
Numerical Simulation and Analysis of External Flow Field of the Van
}

\author{
Kan Zhou ${ }^{1, a}$, Ge Huang ${ }^{1, b}$, Bin Liu, ${ }^{1, c}$, Qi Hu ${ }^{1, d}$ \\ ${ }^{1}$ Institute of Chemical Defense, Beijing, China \\ a517525860@qq.com, blyc61699@163.com, cguyue@sina.com, ${ }^{\text {d } 578125525 @ q q . c o m ~}$
}

\begin{abstract}
This paper uses CFD preprocessing software to build Van model and gridding it, then CFD software is used to simulation the outflow field of Van model, from which the distribution of pressure and velocity is obtained and the outflow field is analyzed. The calculation results indeed reflect the aerodynamic characteristics of the external flow field of the van, and the flow movement on the van surface is better simulated. In addition, the positions where the vortex motion is relatively severe are also found.
\end{abstract}

\section{Introduction}

Aerodynamic performance of an automobile is one of the important performances of an automobile [1], and the outflow field of the body plays a decisive role in this performance. At present, there are two methods to study the outflow field: one is wind tunnel experiment, and the other is numerical simulation by CFD software [2]. Although the wind tunnel experiment can obtain the results with high reliability, it also has the disadvantages of high cost, long period, large equipment, etc., and is vulnerable to the limitations and constraints of wind tunnel boundary conditions (wind speed, wind direction, Reynolds number, etc.). CFD software develops very fast with the continuous improvement of computer technology and numerical technology. CFD software can greatly save financial, manpower, material resources and time needed for the experiment, the application range is wide, can be used for simulating the measured data of experiment is difficult to calculate, to deepen the study of mechanism of turbulent flow problem [3].

At present, CFD technology of computational fluid dynamics has been widely applied in automobile aerodynamics, and has gradually become an important way to study automobile aerodynamics performance, which providing intuitive basis for the selection of aerodynamic shape and modification of modeling in the early stage of new car design [4]. This paper takes a van as the research object, adopts CFD method to carry out numerical simulation of its external flow field, and analyzes its aerodynamic characteristics according to the numerical simulation results.

\section{Governing Equations and Turbulence Models}

The basic laws governing fluid flow are mass conservation law, momentum conservation law and energy conservation law. Consequently, continuum equation, momentum equation and energy equation can be obtained. The N-S equations (Naiver-Stokes equations) obtained by the combination are the general laws governing fluid flow. When applied to aerodynamics, four numerical simulation methods are obtained: panel method, Reynolds average method (RANS), direct numerical simulation method and large eddy simulation method.

Reynolds average method (RANS) is a numerical simulation method widely used in vehicle aerodynamics simulation, which is mainly used to analyze the flow field of the external flow and internal flow. When turbulence pulsation velocity is introduced to average the time of unsteady N-S equations, unknown additional Reynolds stress terms will be generated. At this time, turbulence model should be adopted to solve the closed problem of RANS equations. At present, two-equation model is the most widely used in engineering, and the most basic two-equation model is the Standard $k-\varepsilon$ turbulence model, whose convergence and accuracy meet the requirements of engineering calculation.

Van in the process of driving, the surrounding environment as the air, when the wind speed is less than a third times the speed of sound $(408 \mathrm{~km} / \mathrm{h})$, the air can be thought as incompressible gas, the van speed is far less than the value in the normal speed, so the vehicle can be regarded as incompressible gas surrounding air, so the truck body flow field can be in accordance with the three dimensional isothermal viscous incompressible flow field, does not consider the energy equation [5].

\section{Establishment of Computational Model and Grid Division}

\subsection{Geometric Model}

${ }^{a}$ Corresponding author: 517525860@qq.com 
The transport vehicle model used in the calculation was built in a 1:1 ratio in software. In order to improve the computational efficiency, the creation of complex surfaces such as rearview mirror, exhaust pipe and door handle was neglected in modeling, and the bottom of the model was smoothed(in Fig. 1).

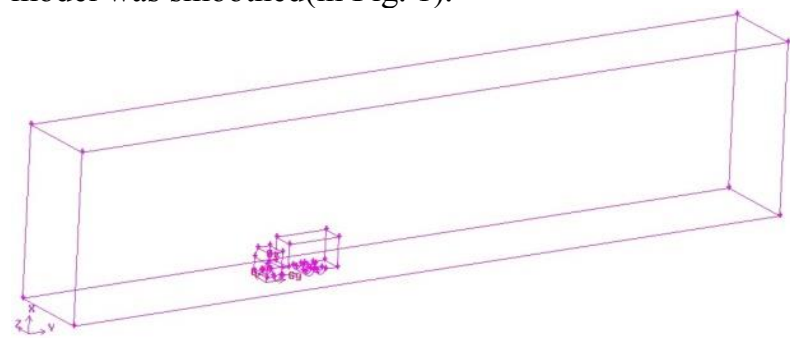

Fig. 1 Van model

\subsection{Establishment of Computing Domain}

The calculation domain is a rectangular area surrounding the Van. For the sake of full development of turbulence, the calculation domain is obtained as follows: the entrance is 3 times the length of the van from the front, the exit is 6 times the length of the van from the rear, the width is 4 times the width of the van, and the height is 5 times the height of the van. The van body size is $\mathrm{Lx} \mathrm{W} x$ $\mathrm{H}$ (length $\mathrm{x}$ width $\mathrm{x}$ height), and the calculation domain size is expressed as 10L x $4 \mathrm{~W} \times 5 \mathrm{H}$ (in Fig. 2).

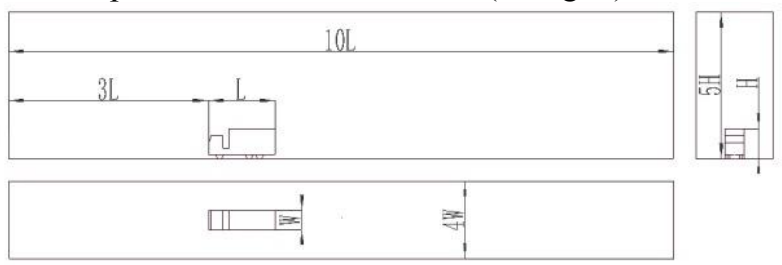

Fig. 2 Computational domain

\subsection{Grid Division}

After determining the geometric model and calculation domain of the transport vehicle, the outer surface of the geometric model and the whole calculation domain need to be meshed. The meshing is very important for the whole calculation process. The quality of the mesh will directly affect the speed, accuracy and convergence of the flow field calculation. In this paper, the calculation domain and model are meshed by CFD preprocessing software Gambit. The mesh is mainly tetrahedral.

In order to solve the problems of complex geometric shape, large computing area, large number of grids and long computing time of transport vehicles, the entire computing domain grid is divided into three layers. The grids close to the van are denser, while those far from the van are relatively sparse, thus improving the calculation accuracy. The total number of grids generated is 5024267 .

\subsection{Boundary Conditions}

Numerical simulation is carried out in a limited computational domain, so each boundary should have specific boundary conditions [6]. The van's speed is generally below $100 \mathrm{~km} / \mathrm{h}$. Simulation of van at a uniform speed of $80 \mathrm{~km} / \mathrm{h}(22.2 \mathrm{~m} / \mathrm{s})$, the car body facing the wind. Boundary conditions are set into 5 categories: inlet boundary, outlet boundary, moving wall surface, fixed wall surface and symmetrical surface boundary conditions. Inlet boundary: take the far front end of the van body as the inlet boundary. When calculating, the given gas velocity is $22.2 \mathrm{~m} / \mathrm{s}$ and the direction is perpendicular to the inlet boundary. Exit boundary: take the far end of the van body as the exit boundary, and calculate the exit boundary condition of the given pressure as the standard atmospheric pressure. The external surface of the van is a fixed wall, which meets the condition of no slippage of the fixed wall. The ground at the bottom is the moving wall surface with the same velocity as the incoming flow. The upper and lateral sides of the outflow field are symmetrical plane boundary.

\subsection{Calculation Method}

Fluent software is used in the solution process, which is widely used in automobile design, oil and gas, aerospace, turbine design and other fields. It can simulate heat and mass transfer, chemical reaction, fluid flow and other complex fluid motion phenomena. It can be used in turbulence, chemical reaction, mixing, heat transfer, rotating flow and shock wave fields.

In this paper, the van is set to travel at a speed of $80 \mathrm{~km} / \mathrm{h}$. At this time, the air flow field is steady and incompressible. Pressure-based coupling algorithm was used to solve the problem. The first-order upwind format was adopted for the discrete format, and the Standard $k-\varepsilon$ model was used. After 119 iterative calculations, continuity residual value was less than $1 \times 10^{-3}$, and the steady-state solution was convergent. The residual curve is shown in Fig. 3.

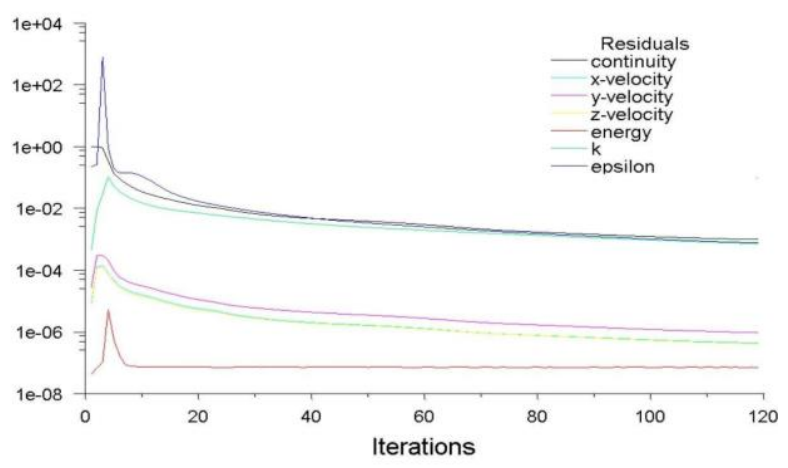

Fig. 3 residual curve

\section{Analysis of Simulation Results}

\subsection{Van Surface Pressure Analysis}

Fig. 4 is the pressure diagram on the symmetrical surface of the van body, ground and flow field. The pressure contour line is marked in the figure.

As can be seen from Fig. 4, the windward surface pressure on the cab of the van is the highest, because the airflow meets the cab and thus is blocked, which greatly reduces the airflow speed. The positive pressure area is formed in the locomotive, and the pressure is between 
50pa and 300pa.After that, the air flows around the side of the cab and above the cab respectively. When flowing through the edge angle of the cab, due to the large curvature of the edge angle, the air flow has no time to turn and local separation occurs, so negative pressure is formed at the edge angle. The pressure line distribution at the side edge angle of the cab is relatively dense compared with the top edge angle of the cab, so the pressure at the side edge angle changes greatly and has a strong pressure gradient. The pressure on the side of the cab ranges from -450 pa to -100 pa.The pressure on the top of cab ranges from -250pa to -100pa.The shape of the top and side of the carriage is relatively smooth, and the negative pressure is increased. The minimum negative pressure at the rear of the carriage is about -50 pa.The pressure in most areas of the outer surface of the whole carriage is maintained between $-100 \mathrm{pa}$ and $-50 \mathrm{pa}$, and the pressure in the position near the cab in front of the carriage is maintained between -150 pa and -100 pa.

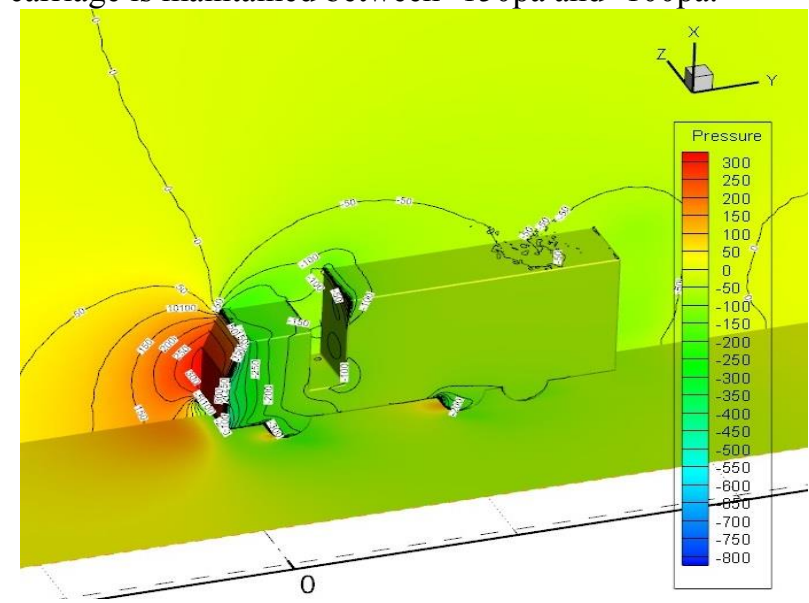

Fig. 4 Distribution of pressure

\subsection{Van Surface Airflow Velocity Analysis}

Fig. 5 is the airflow velocity diagram on the symmetrical surface of the van body, ground and flow field. The isolate of velocity are marked on the surface. As can be seen from Fig. 5, the flow velocity at the side and top edge angle of the cab is large, between $24 \mathrm{~m} / \mathrm{s}$ and $28 \mathrm{~m} / \mathrm{s}$. This is because the larger edge angle is easy to separate the airflow. Another part of the separated airflow is moving toward the rear near the wall, while in the area near the wall the airflow is not separated, resulting from the airflow near the wall of the car body attached to the body, with a low flow rate between $2 \mathrm{~m} / \mathrm{s}$ and $8 \mathrm{~m} / \mathrm{s}$. In regions where vortex structures may be generated, such as the rear of the van, the gap between the rear of the cab and the van, the gas flow rate is low.

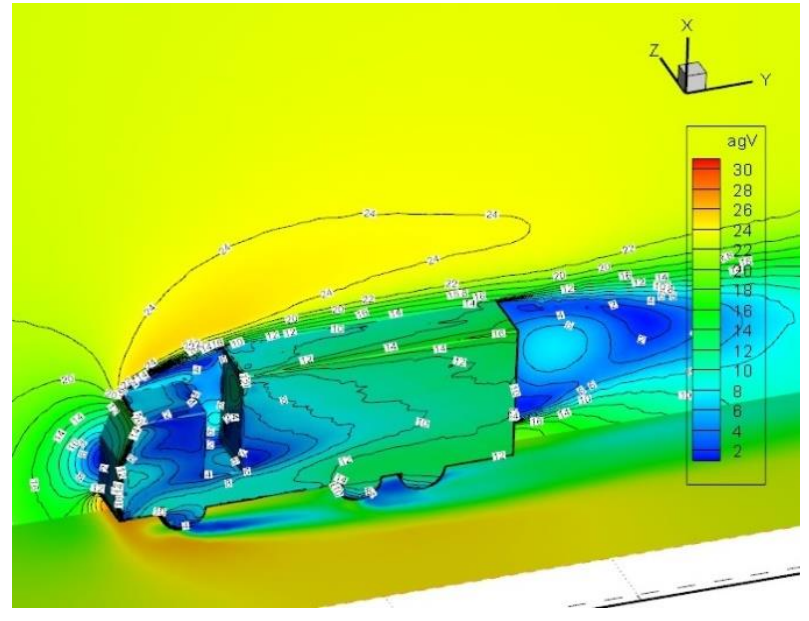

Fig. 5 Distribution of velocity

Relevant studies show that the differential pressure resistance accounts for about $85 \%$ of the total aerodynamic resistance of the van. The pressure difference resistance depends on the size of the wake area of the van. The larger the wake area, the greater the pressure difference resistance, and vice versa. In order to reduce the aerodynamic drag of the transporter and obtain an ideal aerodynamic model, it is necessary to study the motion of the tail scroll. Fig. 6 shows the streamline of the body, ground and flow field symmetrical planes of the van.

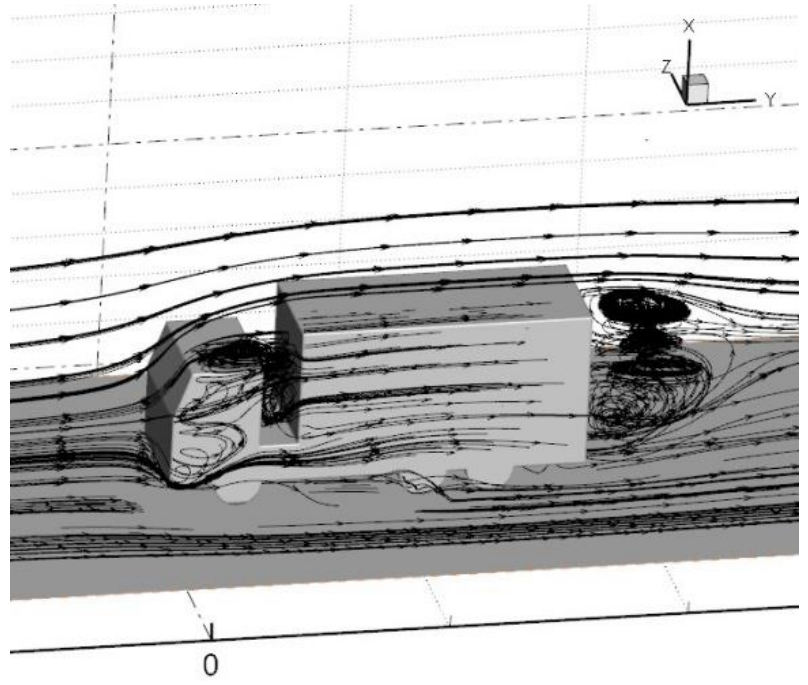

Fig. 6 Distribution of streamline

From Fig. 6, it can be seen that there are many vortices near the rear of the van. These vortices are generated by gas flow gathering behind the van. The airflow flowing through the top of the carriage, side and bottom of the carriage will produce different scale eddies, in which the upper eddies caused by the upside-down of the airflow at the bottom of the carriage and the inner eddies formed by the airflow flowing through the side of the carriage contribute greatly to the induced resistance. These vortices attract each other in the process of flow, and eventually merge into a single pair of large-scale vortices, which exist at a distance from the rear of the car, and gradually become small vortices in the course of motion until they are finally dissipated. In addition, the 
airflow flowing through the side of the cab near the wall rotates upward and moves backward together with the airflow near the roof near the wall. When the cab is blocked, a complex vortex structure is formed in the gap between the cab and the carriage.

\section{Summary}

In this paper, CFD software was used to calculate the three-dimensional flow field of the shape of the van, and the results such as pressure distribution on the surface of the van and velocity distribution of the three-dimensional flow field were obtained. These results were intuitively presented by the graphical function, and the aerodynamic characteristics of the high-speed van were analyzed, and the following conclusions were obtained:

1. The selection of Standard $k-\varepsilon$ turbulence model and the hierarchical division of computational domain grid can greatly reduce the calculation time and relatively ideal calculation results were obtained.

2. The calculation results of the external flow field of the van well simulate the eddy current motion at the corner of the cab edge angle and the tail of the van where there are larger corners, indicating that the eddy current motion at these positions is more intense.

3.The rear modeling of van has a decisive influence on the aerodynamic resistance of the whole van, so it is of great significance to reduce the aerodynamic resistance at the early stage of the new car design by studying the vortex motion of the tail.

Due to the complexity of actual air flow characteristics, there is an inevitable deviation between CFD software and the actual situation in the simulation of the flow field of the van, so in solving the actual aerodynamic problems, wind tunnel test and software simulation should be combined and supplemented to obtain accurate results.

\section{References}

1. Information on http://www.machinedesign.com

2. A. Waschle, The Influence of Rotating Wheels on Vehicle Aerodynamics-Numerical and Experimental Investigation, SAE Paper, 2007-01-0107 (2007)

3. Information on http://www.designnews.com

4. S. Rajneesh, CFD Simulation of NASCAR Racing Car Aerodynamics, SAE Paper, 2008-01-0659 (2008)

5. W. Kieffer, S. Moujaes, N. Armbya, CFD study of section characteristics of Formula Mazda race car wings: submitted to Mathematical and Computer Modeling 43, p. 1275-1287 (2006)

6. CD Adapco Group, Methodology, Computation Dynamics Limited, NY (1999), in press 